\title{
A comparison of software- and hardware-gating techniques applied to near-field antenna measurements
}

\author{
M. M. Leibfritz, M. D. Blech, F. M. Landstorfer, and T. F. Eibert \\ Institute of Radio Frequency Technology (IHF), Universität Stuttgart, Germany
}

\begin{abstract}
It is well-known that antenna measurements are error prone with respect to reflections within an antenna measurements test facility. The influence on near-field (NF) measurements with subsequent NF to far-field (FF) transformation can be significantly reduced applying soft- or hardgating techniques. Hard-gating systems are often used in compact range facilities employing fast PIN-diode switches (Hartmann, 2000) whereas soft-gating systems utilize a network analyzer to gather frequency samples and eliminate objectionable distortions in the time-domain by means of Fourier-transformation techniques. Near-field (NF) antenna measurements are known to be sensitive to various errors concerning the measurement setup as there have to be mentioned the accuracy of the positioner, the measurement instruments or the quality of the anechoic chamber itself. Two different approaches employing soft- and hard-gating techniques are discussed with respect to practical applications. Signal generation for the antenna under test (AUT) is implemented using a newly developed hard-gating system based on digital signal synthesis allowing gate-widths of $250 \mathrm{ps}$ to $10 \mathrm{~ns}$. Measurement results obtained from a YagiUda antenna under test (AUT) and a dual polarized openended waveguide used as probe antenna are presented for the GSM 1800 frequency range.
\end{abstract}

\section{Introduction}

Antenna measurements require free space conditions. However, in practice they are carried out in anechoic chambers equipped with absorbing material. These absorbers still cause reflections although the reflection level is significantly reduced as compared to a non-anechoic test site. They lead to incorrect amplitude and phase values of the acquired electromagnetic fields.

To counteract these errors it is possible to gate out reflected multipath components either by conventional software-

Correspondence to: M. D. Blech

(blech@ihf.uni-stuttgart.de) gating or hardware-gating techniques. Both methods avail themselves of the time difference of arrival (TDOA) of line of sight and multipath signal components. These techniques are presented in Sect. 2.

Multipath propagation errors are mitigated applying gating techniques to measured near-field data. After that a NF to FF transformation is performed and a comparison of results obtained by either gating-technique and an ungated measurement are presented in Sect. 3. It is investigated how marginal errors of maximal $1 \mathrm{~dB}$ of the NF raw data effect the transformed FF as the error propagation through the transformation algorithm is not quite obvious.

The paper closes with a discussion of the measurement results and an evaluation of the novel gating method proposed.

\section{Gating fundamentals}

\subsection{Software-gating}

The software-gating principle is the most commonly applied gating technique. In this technique frequency samples acquired by a vector network analyzer (VNA) are postprocessed by means of a discrete Fourier-transformation (DFT). For this method no special RF measurement instruments are needed. As the postprocessing of the measurement data requires low computational effort an ordinary desktop PC is sufficient.

$N_{\mathrm{M}}$ equidistant frequency samples of the scattering parameter $S_{21}\left(n_{\mathrm{M}} F_{\mathrm{S}}\right)\left(n_{\mathrm{M}}=0,1, . ., N_{\mathrm{M}}-1\right)$ have to be measured over a wide frequency range. This data obtained at positive frequencies has to be zero-padded by at least $N_{\mathrm{M}}-1$ more samples yielding

$S_{21, \text { padded }}\left(n F_{\mathrm{S}}\right)= \begin{cases}S_{21}\left(n F_{\mathrm{S}}\right) & , n \leq N_{\mathrm{M}}-1 \\ 0 & , n>N_{\mathrm{M}}-1\end{cases}$

with $n=0,1, . ., N-1$ before beeing transformed to the discrete analytic time-domain signal $s_{21, \mathrm{a}}\left(k T_{\mathrm{S}}\right)(k=0,1, \ldots$ 


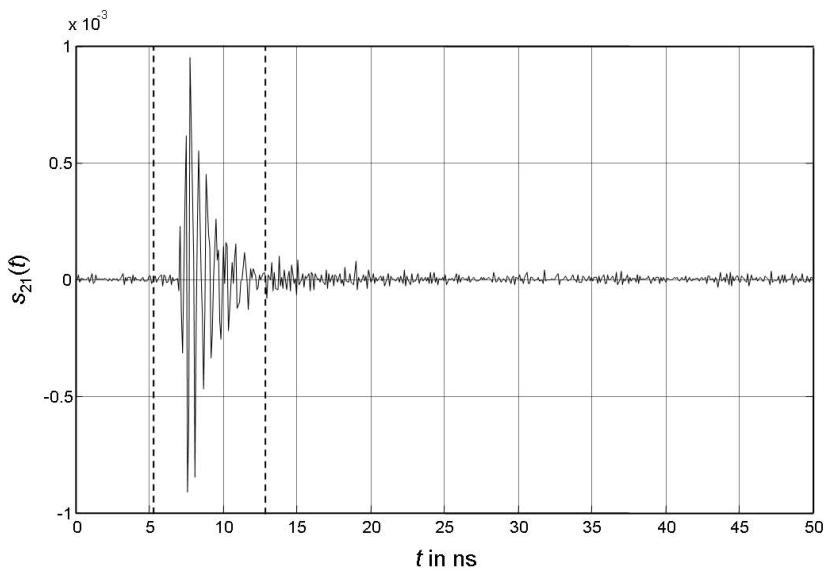

Fig. 1. Impulse response of the transmission link between AUT and probe antenna (black line) and possible time window (dashed lines).

$N-1$ ) (Oppenheim et al., 2004) by the inverse discrete Fourier-transform (IDFT)

$s_{21, \mathrm{a}}\left(k T_{\mathrm{S}}\right)=\frac{2}{N} \sum_{n=0}^{N-1} S_{21, \text { padded }}\left(n F_{\mathrm{S}}\right) \mathrm{e}^{\mathrm{j} \frac{2 \pi}{N} k n}$.

In order to get a fine interpolation in the time-domain signal it is adequate to pad ten times $N_{\mathrm{M}}-1$ ) zeros.

The sampling interval in the frequency-domain is denoted by $F_{\mathrm{S}}$, the time step in the time-domain by $T_{\mathrm{S}}$. It has to be pointed out that the overall bandwidth $B_{\mathrm{LP}}=F_{\mathrm{S}}(N-1)$ of the $N$ discrete frequencies determines the time step $T_{\mathrm{S}}$ between adjacent samples in the time-domain

$T_{\mathrm{S}}=\frac{1}{B_{\mathrm{LP}}}$,

whereas the sampling intervall $F_{\mathrm{S}}$ defines the unambigous range in the time-domain

$T_{\text {unambiguous }}=\frac{1}{F_{\mathrm{S}}}$.

The real-valued impulse response

$s_{21}\left(k T_{\mathrm{S}}\right)=\operatorname{Re}\left\{s_{21, \mathrm{a}}\left(k T_{\mathrm{S}}\right)\right\}$

is expected to be similar to the one depicted in Fig. 1. Signal components which have been received after the main peak due to multipath propagation in the anechoic chamber can be eliminated by applying a time window $w\left(k T_{\mathrm{S}}\right)$ drawn in by dashed lines which gates out the ringing of the impulse

$s_{21, \text { gated }}\left(k T_{\mathrm{S}}\right)=s_{21}\left(k T_{\mathrm{S}}\right) w\left(k T_{\mathrm{S}}\right)$.

This window should have a smooth amplitude tapering in order to avoid leakage effekts in the frequency-domain (Harris, 1978). In the measurements presented here a Hann window has been applied. After that the analytic signal $s_{21 \text {,gated,a }}\left(k T_{\mathrm{S}}\right)$ of the gated signal $s_{21 \text {,gated }}\left(k T_{\mathrm{S}}\right)$ has to be computed by means of discrete Fourier-transform as described in Marple (1999) (see Appendix A) before being transformed back to the frequency-domain by applying the discrete fourier transform (DFT)

$S_{21, \text { gated }}\left(n F_{\mathrm{S}}\right)=\sum_{k=0}^{N-1} s_{21, \text { gated, } \mathrm{a}}\left(k T_{\mathrm{S}}\right) \mathrm{e}^{-\mathrm{j} \frac{2 \pi}{N} n k}$.

This gating procedure has to be applied to all measurement points on the spherical grid before being able to postprocess the data by a NF to FF transformation. The transformation can individually be done for each frequency sample of $S_{21 \text {, gated }}\left(n F_{\mathrm{S}}\right)$.

\subsection{Hardware-Gating}

The principle of the new hardware gating technique developed at the IHF is to evaluate the measured sinusoidal test signal in the time interval after the envelope has reached its steady state and before the first reflected multipath component of the signal occurs. For a typical near-field measurement setup within the GSM 1800 range this is approximately 3-4 ns depending on the settling time of the AUT and the probe, respectively.

In order to evaluate the measured signal properly it is necessary that at least $1.5 \mathrm{~ns}$ of the sinusoidal impulse remain free of multipath components. This time windows corresponds to the interval marked by dashed lines in Fig. 2. As the settling time of the signal transmitted through the bandpass system consisting of both broadband antennas is $4 \mathrm{~ns}$ and the TDOA of the line of sight signal and the first multipath component is approximately $7 \mathrm{~ns}$ (see Fig. 2) there are $3 \mathrm{~ns}$ left for a proper measurement. In this interval the switched carrier has already reached its steady state and multipath components corrupting the received signals have not occured yet.

It should be noted that the bandwidth $B$ of a system and its according rise time $T_{\mathrm{r}}$ are inverse proportional:

$B T_{\mathrm{r}}=C, C=$ const. .

For the acquisition of these short transient signals a fast real time scope or a broadband sampling scope is needed. With a sampling scope a higher dynamic range as well as a better resolution can be obtained.

For the evaluation of the envelope of a received signal a CW-signal with a rise time in the sub-ns region is required. This is necessary to ensure that the sinusoidal carrier has reached its steady state before multi-path components corrupt the signal. PIN-diode switches which are commonly used to gate out objectionable signal components in far-field measurement setups (Hartmann, 2000) cannot be used here as they are not available with a switching time below $1 \mathrm{~ns}$. Therefore a custom signal generator was developed (Leibfritz, 2006) that synthesizes a sinusoidal impulse 

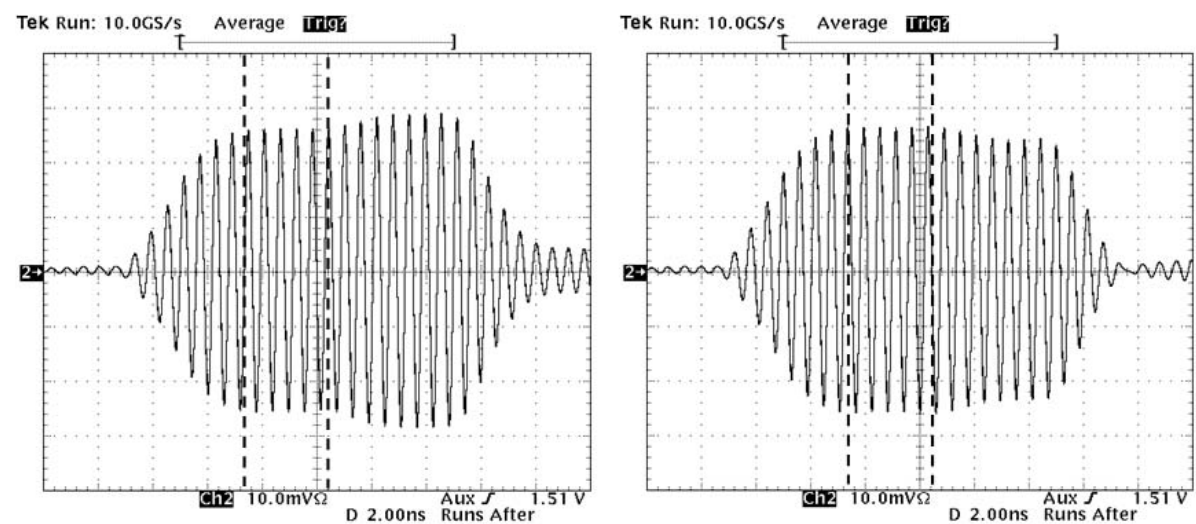

Fig. 2. Constructive (left) and destructive (right) interference caused by multipath propagation in the anechoic chamber and possible time window.

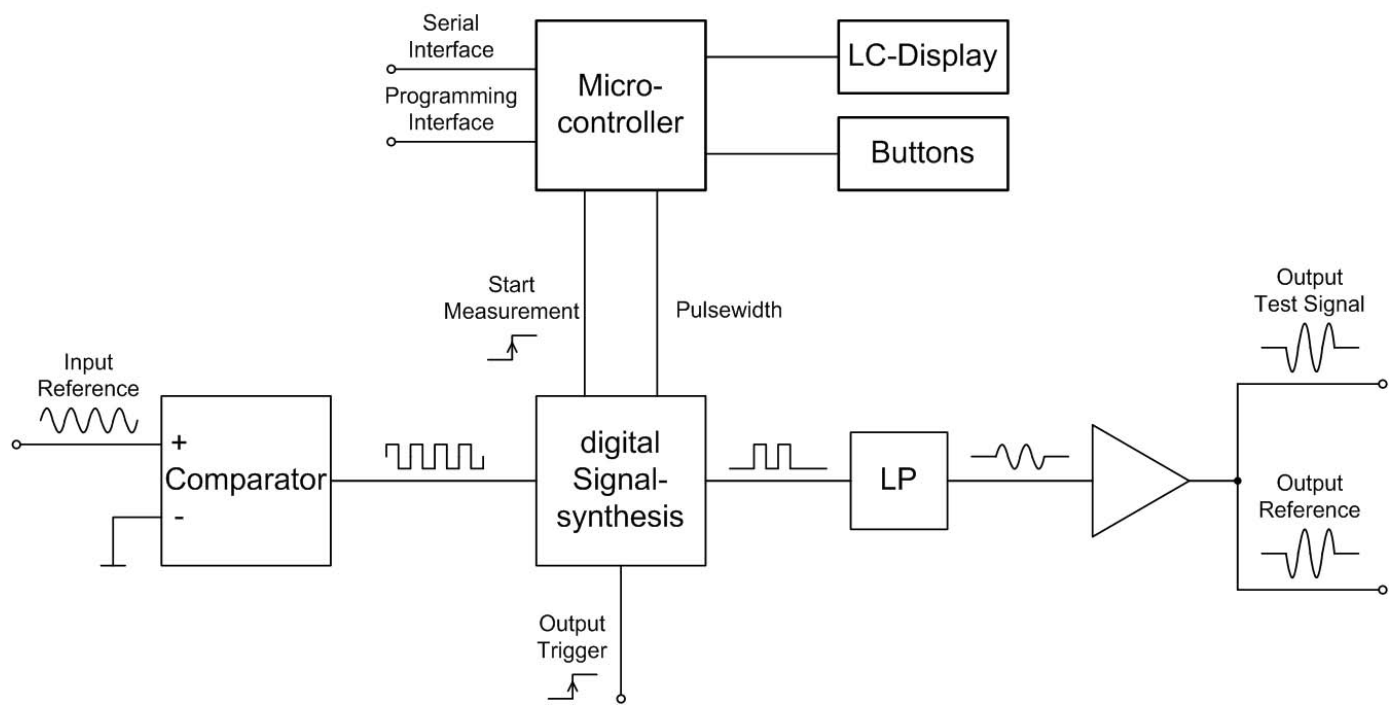

Fig. 3. Block diagram of the impulse generator employed for hardgating measurements.

by means of digital signal processing and subsequent low pass filtering. In this way a rise time of $150 \mathrm{ps}$ is obtained. The block diagram including a microcontroller based interface and a broadband gain stage providing an output level of $12 \mathrm{dBm}$ is depicted in Fig. 3.

\section{Measurements}

To evaluate the influence of the proposed gating techniques on the quality of a measured radiation pattern of an AUT a broadband two element yagi antenna is taken as test antenna (Fig. 4). This custom-made antenna has a center frequency of $1.7 \mathrm{GHz}$, a $10 \mathrm{~dB}$ bandwidth of approximately $500 \mathrm{MHz}$ and thus exhibits a suitable rise time of the envelope for the hardgating system. It is fed by a coaxial $\frac{\lambda}{4}$-balun and its boom is made of polyvinyl chloride (PVC).
The size of this single test antenna allows far field measurements in a distance of a few meteres, but very often much larger arrays like base station antennas have to be measured requiring a minimum distance of more than $10 \mathrm{~m}$ to ensure far-field conditions. This and the fact that decreasing measurement frequencies result in an increasing distance of the Fraunhofer-region lead to near-field measurements as the preferred universal measurement technique. Reference measurements are carried out on an optimized GSM 1800 test facility of a well known manufacturer of mobile phones. Formerly a division of Siemens. These measurement results are assumed to be error-free as the probe is mounted on an arc orbiting around the AUT causing hardly any distortions of the measurement signals. Thus all measurements namely an ungated measurement in the frequencydomain and a soft- as well as a hard-gated measurement carried out in the anechoic chamber of the Institute of Radio 


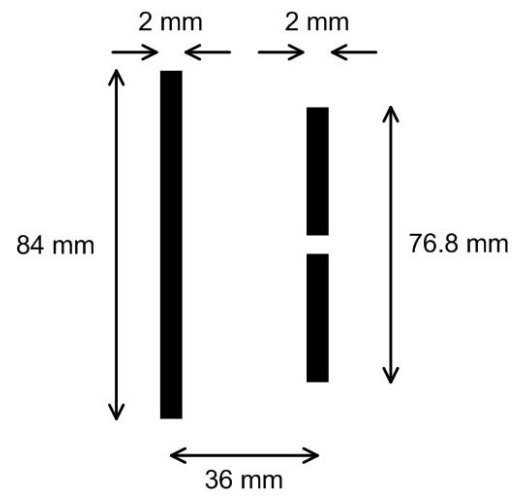

Fig. 4. Dimensions of the investigated AUT.

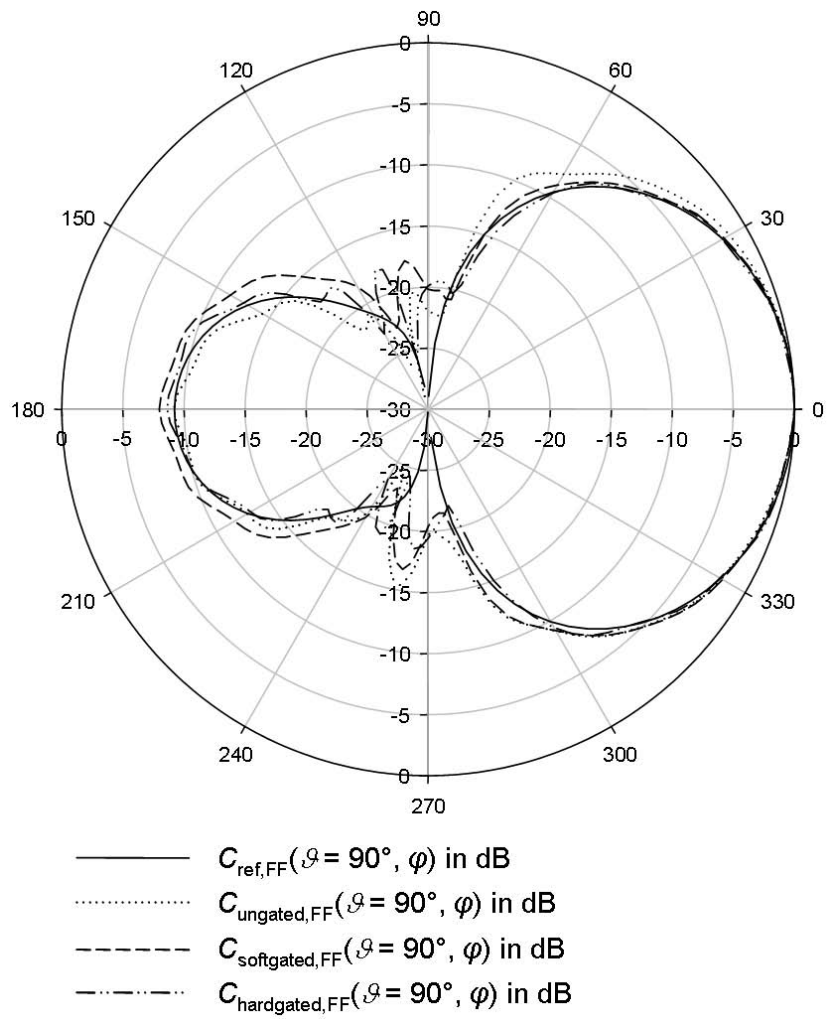

Fig. 5. Computed far-field patterns of the AUT (E-plane).

Frequency Technology at the Universität Stuttgart are compared to them. All frequency-domain measurements conducted there are made with a Wiltron 360B VNA. The timedomain signal acquisition of the hardgating system is carried out by a Tektronix TDS694C real time scope.

The spherical electric near-field is measured with an elevation over azimuth positioner from Orbit and a custom-made dual polarized open-ended waveguide used as probe antenna. This dual polarized probe helps to save time for the longlasting measurements as it does not have to be positioned and all data can be acquired in one run. The probe antenna

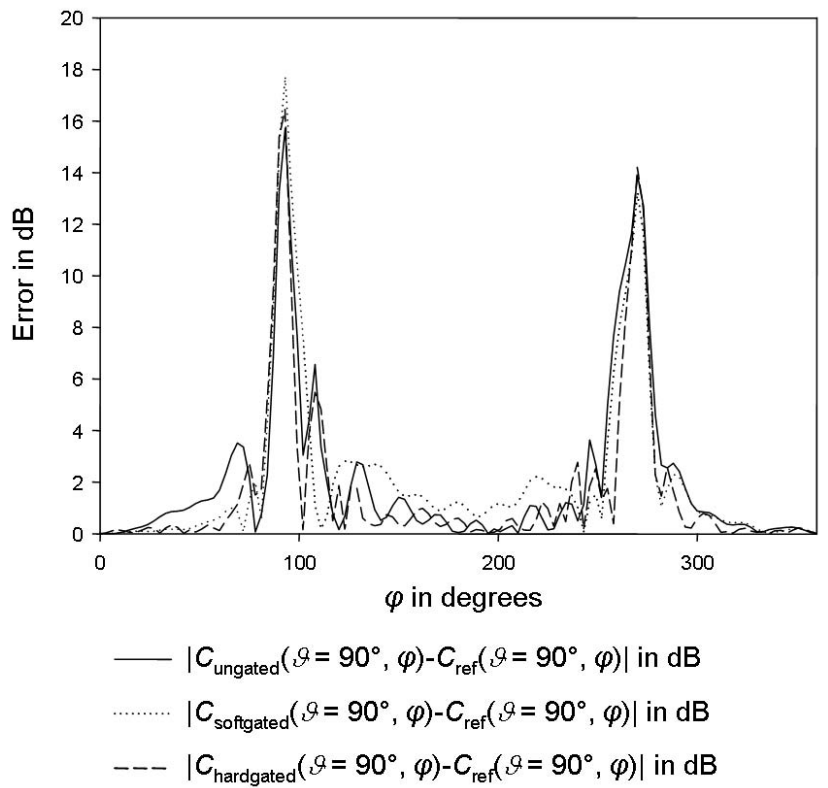

Fig. 6. Absolute error of computed far-field patterns.

is mounted at a distance of $1.7 \mathrm{~m}$ from the AUT whereas the angular measurement grid is $3^{\circ}$ in $\vartheta$ and $\varphi$.

After the gating procedure the near-field data is transformed to the far field by an algorithm (Christ, 1995) also developed at the Universität Stuttgart using first order probe correction (Hansen, 1988).

As the near-field to far-field transformation algorithm is sensitive to amplitude and phase the near-field data should be acquired as accurate as possible. Otherwise small NF errors can lead to even worse distortions in the FF transormation results. Figure 5 shows the measured and computed far-field radiation patterns of the AUT in the E-plane. In Fig. 6 the absolute error of the computed far-field in the E-plane is shown. It can be seen that the error in the near- and far-field has its maximum close to the zeros of the reference measurement pattern. Because of this error peak of almost $20 \mathrm{~dB}$ this cannot be caused by the measurement instruments themselves even if the signals were slightly above the noise floor the measurement error cannot be more than $6 \mathrm{~dB}$. In fact these errors arise from the elevation positioner the AUT is attached to which has a diameter of $30 \mathrm{~cm}$. Hence the AUT suffers from the shadowing effect due to the dimensions of this obstacle when it moves in between the yagi and the probe. In this case neither of both gating techniques can improve the measurements, because the positioner is placed right in the line of sight between the two antennas and only reflected non line of sight components exist. The influence of the azimuth positioner can be neglected as it is located $2.5 \mathrm{~m}$ below the AUT and completely covered with absorbers.

Therefore further error analysis is only carried out for the main beam. In Fig. 6 no distinct improvement by either of 
Table 1. Absolute mean error of ungated and gated far-field patterns after NF to FF transformation.

\begin{tabular}{cccc}
\hline Angular Range & Measurement & $|\bar{E}|(\mathrm{NF})$ & $|\bar{E}|(\mathrm{FF})$ \\
\hline$-60^{\circ} . .+60^{\circ}$ & ungated & $0.42 \mathrm{~dB}$ & $0.49 \mathrm{~dB}$ \\
$-60^{\circ} . .+60^{\circ}$ & software-gating & $0.35 \mathrm{~dB}$ & $0.25 \mathrm{~dB}$ \\
$-60^{\circ} . .+60^{\circ}$ & hardware-gating & $0.42 \mathrm{~dB}$ & $0.17 \mathrm{~dB}$ \\
\hline$-75^{\circ} . .+75^{\circ}$ & ungated & $1.10 \mathrm{~dB}$ & $0.88 \mathrm{~dB}$ \\
$-75^{\circ} . .+75^{\circ}$ & software-gating & $1.12 \mathrm{~dB}$ & $0.46 \mathrm{~dB}$ \\
$-75^{\circ} . .+75^{\circ}$ & hardware-gating & $1.10 \mathrm{~dB}$ & $0.38 \mathrm{~dB}$ \\
\hline
\end{tabular}

Table 2. Absolute mean error of ungated and hardgated FF measurements.

\begin{tabular}{ccc}
\hline Angular Range & Measurement & $|\bar{E}|(\mathrm{FF})$ \\
\hline$-60^{\circ} . .+60^{\circ}$ & ungated & $0.25 \mathrm{~dB}$ \\
$-60^{\circ} . .+60^{\circ}$ & hardware-gating & $0.21 \mathrm{~dB}$ \\
\hline$-75^{\circ} . .+75^{\circ}$ & ungated & $0.28 \mathrm{~dB}$ \\
$-75^{\circ} . .+75^{\circ}$ & hardware-gating & $0.25 \mathrm{~dB}$ \\
\hline $0^{\circ} . .360^{\circ}$ & ungated & $0.93 \mathrm{~dB}$ \\
$0^{\circ} . .360^{\circ}$ & hardware-gating & $0.60 \mathrm{~dB}$ \\
\hline
\end{tabular}

the gating techniques can be seen. Therefore in the following the absolute mean error (9) between ungated, soft- and hardgated measurements and reference data is evaluated in order to evaluate the different measurement results,

$|\bar{E}|=\frac{\sum_{n=N_{\text {Start }}}^{N_{\text {Stop }}}\left|C(n \Delta \varphi)-C_{\text {ref }}(n \Delta \varphi)\right|}{\left(N_{\text {Start }}-N_{\text {Stop }}+1\right)}$

where $\Delta \varphi$ is the angle increment of the measurement grid. $N_{\text {Start }}$ and $N_{\text {Stop }}$ are the indices of of the first and the last sample in the regarded interval, respectively. $C_{\text {ref }}(n \Delta \varphi)$ is the radiation characteristic taken as reference whereas $C(n \Delta \varphi)$ is the radiation characteristic being erroneous.

As described earlier it is only reasonable to evaluate the main lobe of the AUT. For the comparison shown in Table 1 the maximum angular range of the radiation pattern of the two element yagi in the E-plane is limited to $\pm 75^{\circ}$. As can be seen there the absolute value is evaluated in the near-field as well as in the far-field. The absolute mean error is reduced by both software- and hardware-gating techniques. Regarding the main lobe one can note that the ungated measurement yields a half-power beam width which has an error of $5^{\circ}$ whereas the gated measurements coincide with the reference pattern.

The influence of the roll axis of the positioner can be shown if this axis is removed and a far-field pattern of the E-plane of the AUT is measured with the azimuth rotor only.

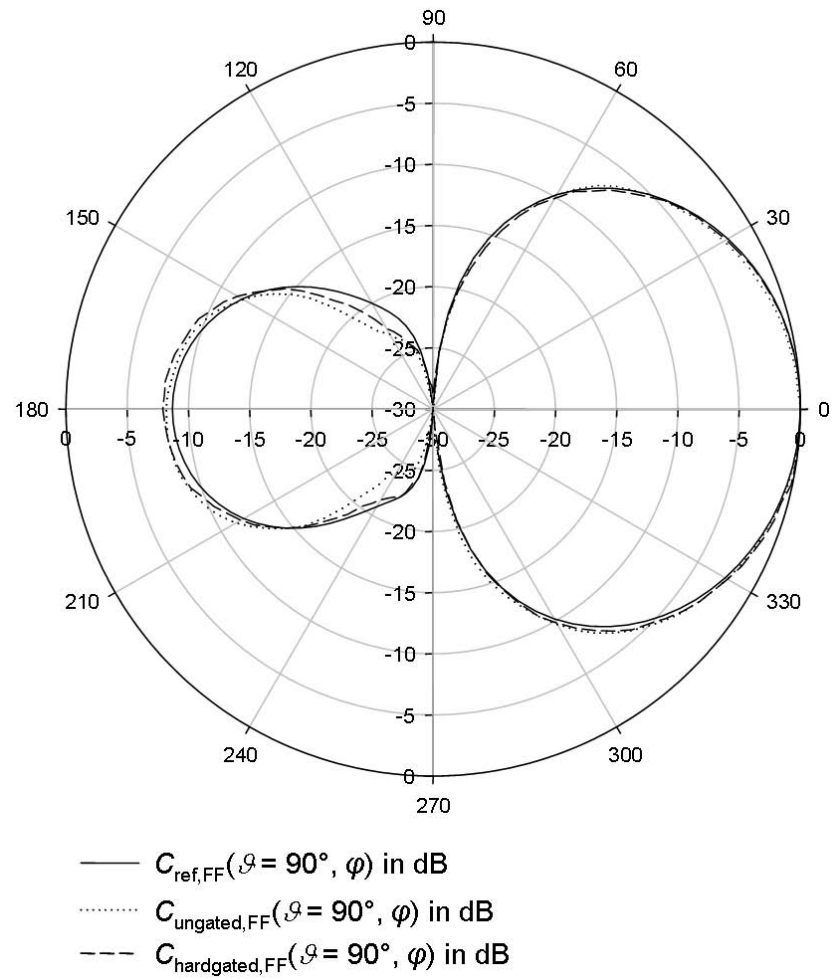

Fig. 7. Measured far-field patterns of the AUT (E-plane).

Then the distortions caused by the positioner should vanish. The corresponding patterns for an ungated and a hardgated far-field measurement are compared to the reference pattern determined earlier (Fig. 7). It shows that there are no major distortions in the vicinity of the zeros any more. So the sidelobes in the transformed far-field are traced back to the incorrect near-field in some regions of the sphere caused by the disadvantageous dimension of the roll axis as proposed. The resulting absolute errors of this far-field measurement are plotted in Fig. 8. Table 2 shows a comparison of the absolute mean error. In this case an evaluation of the whole circular range is possible, significantly reducing the errorlevel as compared to the ungated measurement.

\section{Conclusions}

The influence of software- and hardware-gating techniques on erroneous near-field measurements data and subsequent near- to far-field transformation have been investigated. It has been shown that the resulting main lobe accuracy of the far-field pattern in the E-plane of a two element yagi antenna used as AUT can be improved by $0.5 \mathrm{~dB}$ using the novel hardware-gating system.

For a far-field measurement the improvement in the whole E-plane is $0.33 \mathrm{~dB}$ as compared to the reference pattern.

Further investigations will have to be carried out using UWB antennas as well as an elevation positioner with 


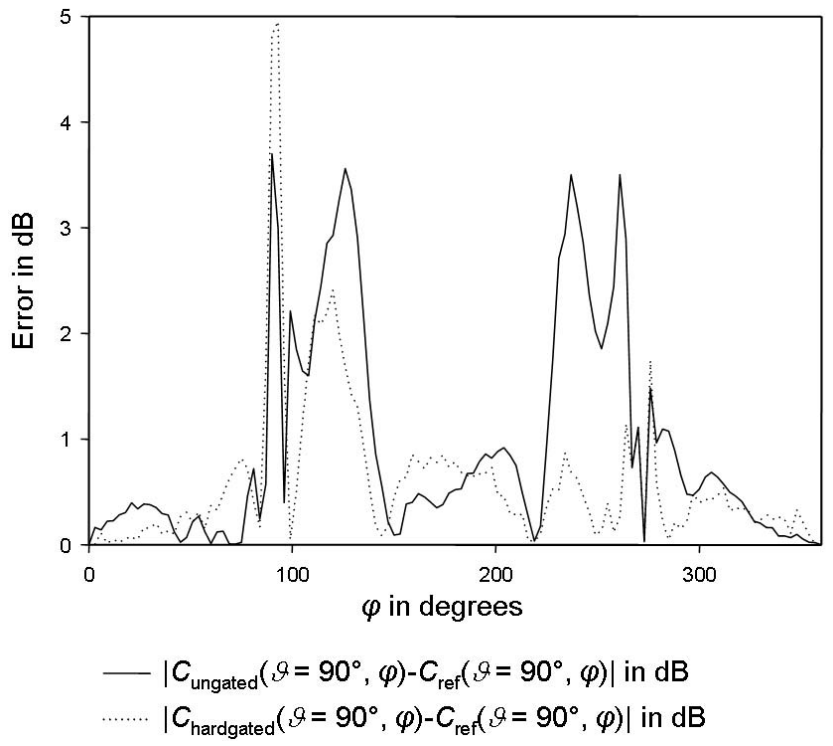

Fig. 8. Absolute error of measured far-field patterns.

reduced size. Employing state of the art measurement instruments with higher dynamic range than the ones used for the measurements presented here is supposed to lead to even better results.

\section{Appendix A}

\section{Computation of the discrete time analytic signal}

In Sect. 2.1 it is necessary to compute the discrete time analytic signal of the time-domain signal $s_{21 \text {,gated }}\left(k T_{\mathrm{S}}\right)$ in order to obtain a one sided spectrum. Therefor $S_{\text {aux, } 1}\left(n F_{\mathrm{S}}\right)$, the DFT of $s_{21, \text { gated }}\left(k T_{\mathrm{S}}\right)$, is computed in a first step

$S_{\text {aux }, 1}\left(n F_{\mathrm{S}}\right)=\operatorname{DFT}\left\{s_{21, \text { gated }}\left(k T_{\mathrm{S}}\right)\right\}$.

Then the auxiliary signal $S_{\text {aux,2 }}\left(n F_{\mathrm{S}}\right)$ has to be created according to

$S_{\text {aux }, 2}\left(n F_{\mathrm{S}}\right)= \begin{cases}S_{\text {aux }, 1}(0) & , n=0 \\ 2 S_{\text {aux }, 1}\left(n F_{\mathrm{S}}\right) & , 1 \leq n \leq \frac{N}{2}-1 \\ S_{\text {aux }, 1}\left(\frac{N}{2} F_{\mathrm{S}}\right) & , n=\frac{N}{2} \\ 0 & , \frac{N}{2}+1 \leq n \leq N-1 .\end{cases}$

In a final step the analytic time-domain signal $s_{21 \text {,gated,a }}\left(k T_{\mathrm{S}}\right)$ is obtained by transforming $S_{\text {aux }, 2}\left(n F_{\mathrm{S}}\right)$ back to the time-domain by applying the IDFT

$s_{21, \text { gated,a }}\left(k T_{\mathrm{S}}\right)=\operatorname{IDFT}\left\{S_{\text {aux }, 2}\left(n F_{\mathrm{S}}\right)\right\}$.

The original time-domain signal $s_{21 \text {,gated }}\left(k T_{\mathrm{S}}\right)$ can be obtained by taking the real part of $s_{21 \text {,gated,a }}\left(k T_{\mathrm{S}}\right)$, whereas the absolute value of $s_{21 \text {,gated, a }}\left(k T_{\mathrm{S}}\right)$ provides its envelope.
Acknowledgements. The authors wish to thank the Hittite Microwave Corporation and ON Semiconductor for providing samples of their integrated circuits for the development of the hardgating system.

\section{References}

Christ, J.: Korrektur prinzipbedingter und durch die Meßumgebung verursachter Fehler bei der Nahfeld-Fernfeld-Transformation, Dissertation, Universität Stuttgart, Shaker-Verlag, Aachen, 1995.

Hansen, J. E.: Spherical Near-Field Antenna Measurements, Institution of Electrical Engineers, London, 1988.

Harris, F. J.: On the Use of Windows for Harmonic Analysis with the Discrete Fourier Transform, Proceedings of the IEEE, vol. 66, no. 1, January, 1978.

Hartmann, J.: Grenzen der Störstrahlungsunterdrückung bei der Vermessung von Mikrowellen- und Millimeterwellenantennen in kompensierten Doppelspiegel-Compact-Range-Meßanlagen, Dissertation, Universität der Bundeswehr, München, 2000.

Leibfritz, M. M.: Effiziente Methoden zur Diagnose bei Gruppenantennen unter Verwendung der sphärischen Nahfeldmesstechnik, Fortschrittsbericht, Universität Stuttgart, 2006.

Marple Jr., S. L.: Computing the Discrete-Time "Analytic" Signal via FFT, IEEE Transactions on Signal Processing, vol. 47, no. 9, September, 1999.

Oppenheim, A. V., Schafer, R. W., and Buck, J. R.: Zeitdiskrete Signalverarbeitung, Pearson Studium, München, 2004. 